\title{
An advanced MPPT scheme for standalone solar powered PV system using neurofuzzy estimator based on measured data
}

\author{
Ferdaws Ben Naceur*, Abdelaziz Salah Saidi**, ***, ****, Javed Khan Bhutto** and \\ Mohamed Ali Mahjoub* \\ * University of Sousse, ISITCom, 4011, Sousse, University of Sousse, National Engineering School of Sousse, LATIS - Laboratory of \\ Advanced Technology and Intelligent Systems, 4023, Sousse, Tunisia. \\ ** Department of Electrical Engineering, King Khalid University, Abha., Abha City, P.C.: 61411, Al-Quri-Qer Quarter. Saudi Arabia. \\ ***Université de Tunis El Manar, Ecole Nationale d'Ingénieurs de Tunis, Laboratoire des Systèmes Electriques. \\ **** Corresponding Author: asaidi@kku.edu.sa
}

Submitted : 11/12/2020

Revised :06/09/2021

Accepted : 19/09/2021

\begin{abstract}
This paper deals with the problem of the optimization of the power, delivered by the photovoltaic panel (PVP). To achieve this aim, a neurofuzzy estimator (NFE), followed by a conversion coefficient and a calculation stage of the optimal duty cycle, has been developed. The NFE is used to calculate the open circuit voltage corresponding to each solar radiation, based only on the standard open circuit voltage. A coefficient, determining for each climatic condition the voltage of the maximum power directly from the open circuit voltage, is estimated by a measured test. Finally, the optimal duty cycles are, next, determined by the input/output equation of boost converter. The system performance, under different scenarios, has been checked carrying out MATLAB simulations, using an existing photovoltaic model and real weather data, and comparing the simulation results with the measured one. The results demonstrate the effectiveness of the present approach. The efficiency of the proposal maximum power point tracking (MPPT) is proved, and it showed that this controller can generate almost $99 \%$ of the real PVP maximum power.
\end{abstract}

Keywords: Photovoltaic system; MPPT controller; Neuro-Fuzzy; Solar radiation sensor.

\section{INTRODUCTION}

A photovoltaic generator has V-I characteristics, which are nonlinear in nature and vary with irradiation and temperature. A specific operating point on V-I and Power-Volt curves is determined using maximum power point (MPP) tracking. At this unique point on the curves, the PV panel will produce the maximum output power at maximum efficiency. By using various MPPT techniques and algorithms, we can arrange the point at that level where to get maximum power irrespective of the irradiation level. 
Consequently, for the same solar radiation, the power delivered will be different according to the load. An MPPT controller can, therefore, be used to control the converter connecting to the load (for example a battery) and the photovoltaic panel in order to supply continuous maximum power to the load.

Many research and study have been developed previously, and the MPPT has been used well in various applications (Mahmoud et al., 2020; Salman et al., 2018; Sellami et al., 2018; Saidi et al., 2018). Recently, a new technique for current-sensor'-less MPPT in single phase, grid-connected voltage source inverters (VSI) in photovoltaic, is suggested in (Dousoky and Masahito, 2017) by only using the voltage parameters to eliminate the current sensor. To achieve this, a new parameter is defined for power-angle-based MPPT. The MPPT algorithm for grid-connected Photo-Voltaic system has been proposed in (Mohammed et al., 2020; Saidi and Helmy, 2019; Lulian and Antoneta, 2015). In this method, an extremum is obtained as a dithering signal obtained from the DC-link voltage ripple. The obtained extremum controls the proposed MPPT technique for grid-connected Photovoltaic systems, without intermediate DC-DC converter stage. A comparative study between with and without MPPT for Photovoltaic panels has been presented (Sachin and Vivek, 2016; Omnia et al., 2020) for the experimental charging of energy storage devices like battery and the ultra-capacitor. MPPT techniques such as P\&O, In Con., and Hill-climbing are compared in (Dandoussou et al., 2016). The energy performance and general costs issues for some of the MPPT algorithms used in Photo-Voltaic applications have been compared in (Osisioma et al., 2017).

A considerable work has been developed in modeling and simulating photovoltaic (PV) systems especially on sizing, like (Ferdaws et al., 2020) who used an ANFIS estimation algorithm in order to estimate a data base of instantaneous photovoltaic power for sizing a PVP/Battery power station and (Nouhaila et al., 2021) who made a sizing of a standalone PV/battery/hydrogen hybrid system using the genetic algorithm (GA).

Other works have been dedicated to the optimization of PV systems, such as (Ferdaws et al., 2021) who used a deep learning algorithm to estimate the climatic factors of a PV system connected to the grid in order to compensate the energy price.

Different research work has been focused on the maximization of the energy output of the PV arrays, like (Abdelaziz et al., 2021) who implement the maximum power point tracking system for the Photo-Voltaic panel based on an estimation artificial NN (ANN) algorithm and (Avila et al., 2020) who used deep reinforcement learning (DRL) techniques to address the MPPT problem of a PV array under partial shading conditions.

The study presents an understanding on the aspects that should be well-thought-out while selecting a suitable technique for a specific application. The performances and the anomalies related with several stage inverter architectures used for the prevalent MPPT techniques have been investigated in (Jiang et al., 2017). A new modified MPPT algorithm has been carried out to instantly trace the unique point of the Photo-voltaic arrays. The modified algorithm has been carried out in a Single-phase, Two-stage photo-voltaic converter in which the DC sensors are eliminated. Based on the relation between the variation of the Open-circuit voltage (VOC) and the Short- circuit current (ISC) along with the perturb and observe algorithm, an MPPT technique has been proposed in (Rahmani et al., 2015). The proposed MPPT technique constitutes a control structure, which contains three loops. These loops are referred to as E- loop, R-loop, and S-loop MPP. These loops are based on these three considerations: (i) voltagecurrent relation of PV that provides MPP, (ii) the duty ratio of the converter to fix operating point at MPP, and (iii) technique to evaluate the changing meteorological conditions and the limit criterion to calculate its constant meteorological conditions. To interface PV systems with a power grid, an MPPT method along with an innovative generalized power quality conditioning system has been presented in (Abdelaziz, 2020). The interfacing system consists of an improved series active filter for compensation even with nonlinear unbalanced load conditions. It is designed to produce the $\mathrm{o} / \mathrm{p}$ voltages for compensation of the source voltage deficits, highly suppressing the grid and current harmonics. In this paper, a new method is developed to determine, in real time, the optimal duty cycle used 
to operate the PVP in its maximum power in climatic parameters. The idea is to develop a neurofuzzy estimator that can determine the PVP open circuit voltage, based on the instantaneous climatic condition. A new relationship between the voltage of the open circuit and the voltage corresponding to the maximum power has been established to determine the optimal duty cycle. This optimal duty cycle is used to control the boost converter to make the PVP operate in its maximum power.

In the first part of this paper, a description of the proposed MPPT controller and a modeling of the structure, which consists of a photovoltaic generator coupled through an adapter DC/DC converter to a DC load, are presented. In the next part of the paper, the simulation results are provided, in order to demonstrate the effectiveness of the proposed MPPT controller. Finally, the conclusion section summarizes the effectiveness and validity of proposed neural network estimator system and also provides the comparison of various MPP techniques.

\section{DESCRIPTION OF THE APPROACH}

A novel MPPT controller strategy, coupled with a real time accurate estimate of the optimum duty cycle to operate the PVP at its maximum power through dc-dc converter, has been evolved with the following objectives:

- To maximize the power delivered by the PVP.

- To develop a control scheme using a sensor (solar radiation and temperature sensor).

- To implement a simple controller with only one input and one output.

- To optimize more than two different PVPs in a single application.

Figure 1 represents the proposed block diagram of PV model that consists of PV Panel, Sensors, Boost converter, MPPT controller, and DC Load.

The figure shown in the power converter stage connects the panel to the DC load. The power converter stage consists of DC-DC converter, which is connected to the output filter and the MPPT control unit. The control unit detects maximum power at a particular point $\left(\mathrm{P}_{\max }\right)$ of Photo-Voltaic systems. The MPPT drives the operating point of the solar panel to extract the maximum power.

The control unit optimizes the duty cycle in real time to control the boost converter and make the PVP operate in it maximum power for varying solar condition. With steady state operation, the transfer function for boost converter is deduced as follows:

$V_{L}=\frac{V_{P V}}{1-\alpha}$

where $\alpha$ is the Duty Ratio/cycle of DC-DC Converter evaluated from the control unit, $\mathrm{V}_{\mathrm{L}}$ is the converter's voltage output, and $\mathrm{VPV}_{\mathrm{PV}}$ is the voltage output of the panel.

The emphasis of proposed approach is to achieve that optimal point (MPP) by analyzing the insolation value G from PV panel for a day by controlling the boost converter. 


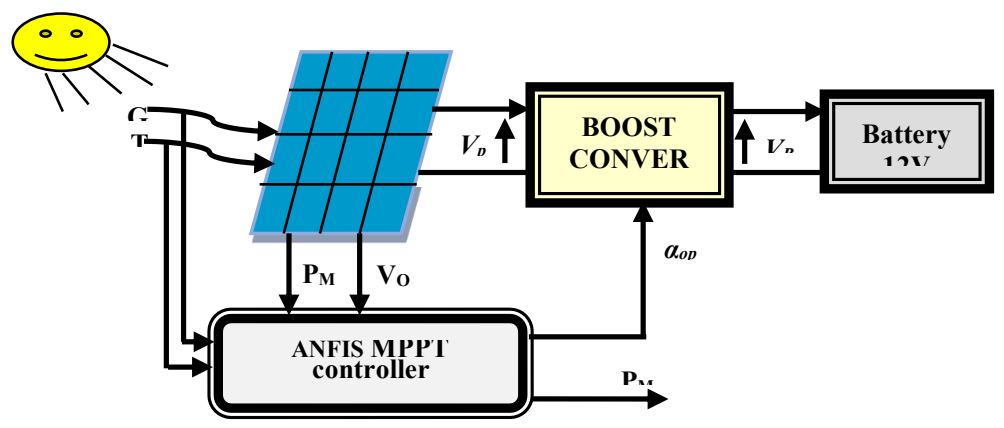

Figure 1. PV system with MPPT controller.

Figure 2 presents the MPPT algorithm framework, which details the design of the algorithm according to four steps.

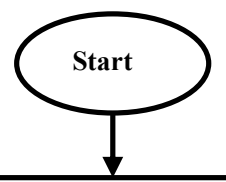

Read the PV electrical parameter: solar radiation G, temperature $T$, standard open circuit voltage $V_{o c-s}$, and the load voltage $V_{L}$. Where $V_{o c-s}=19.8 \mathrm{~V}$ and $V_{L}=V_{m} /\left(1-\alpha_{o p}\right)$

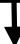

- $\quad$ Read the open circuit voltage $V_{O C}$

- Calculate the maximum voltage $\mathrm{Vm}$ expressed by: $\mathrm{Vm}=\mathrm{V}_{\mathrm{oc}} / \mathbf{1 . 2}$.

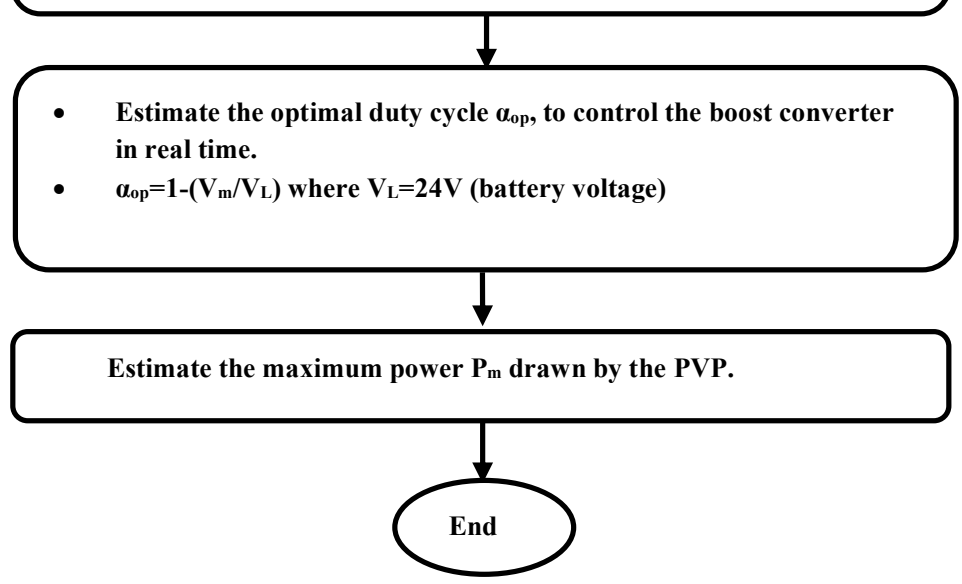

Figure 2. MPPT algorithm framework. 


\section{PHOTOVOLTAIC PANEL}

Figure 3 represents the equivalent electrical circuit of $\mathrm{PV}$ cell as a diode that is connected in parallel with current source.

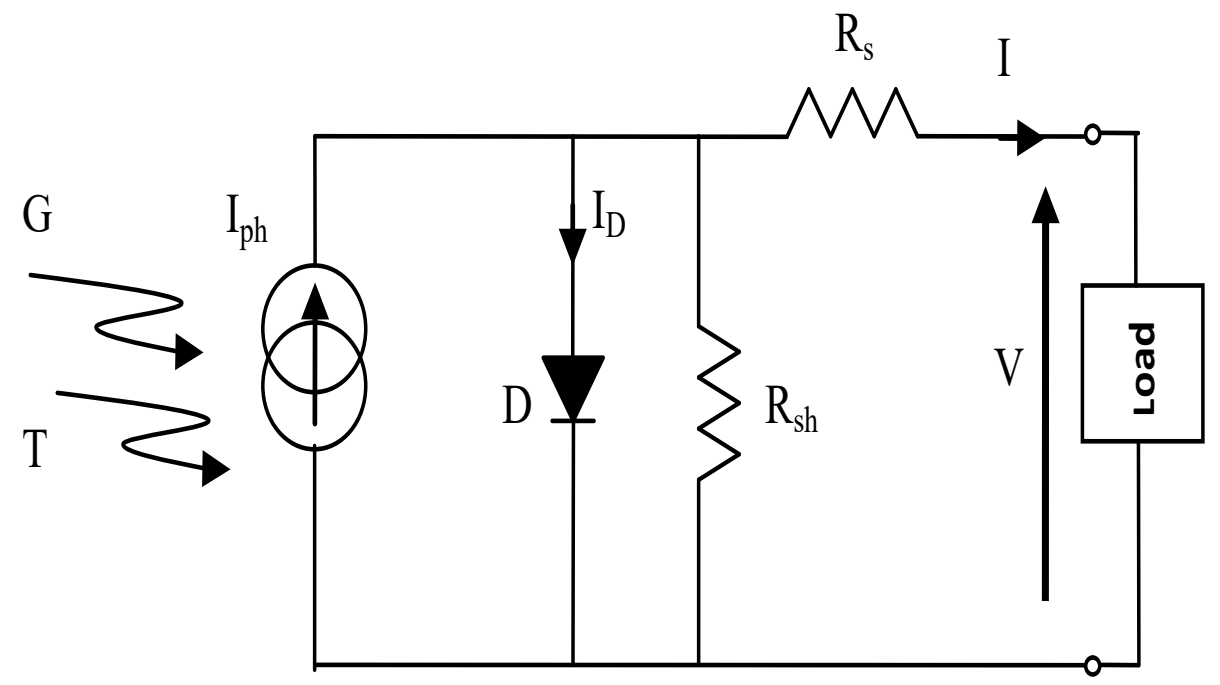

Figure 3. Generalized electrical circuit of Photo-Voltaic cell. follows:

According to the Figure 3, the relationship between the current and the voltage in a single diode is expressed as

$I=I_{p h}-I_{S}\left[\exp \left(\frac{q\left(V+I R_{S}\right)}{k T A}\right)-1\right]-\frac{V+I R_{S}}{R_{S h}}$

The photocurrent Iph is given by the following equation:

$I_{p h}=\frac{G}{G_{r e f}}\left[I_{p h, r e f}+c_{T}\left(T-T_{r}\right)\right]$

where $\mathrm{G}$ is insolation, and $\mathrm{T}$ is the temperature of the cell in degree Kelvin.

The Reverse saturation current $\left(\mathrm{I}_{\mathrm{rs}}\right)$ of the cell is dependent on the PV cell temperature $\mathrm{T}$ and expressed as follows:

$I_{S}=I_{s, r e f}\left[\frac{T}{T_{\text {ref }}}\right]^{3} \exp \left(\frac{q E_{G}}{k A}\left[\frac{1}{T_{\text {ref }}}-\frac{1}{T}\right]\right)$

To form an array, these PV cells are in series and parallel y connected. All PV cells connected in array are considered to have the same characteristics. The equivalent circuit of PV array is presented in Figure 4. 


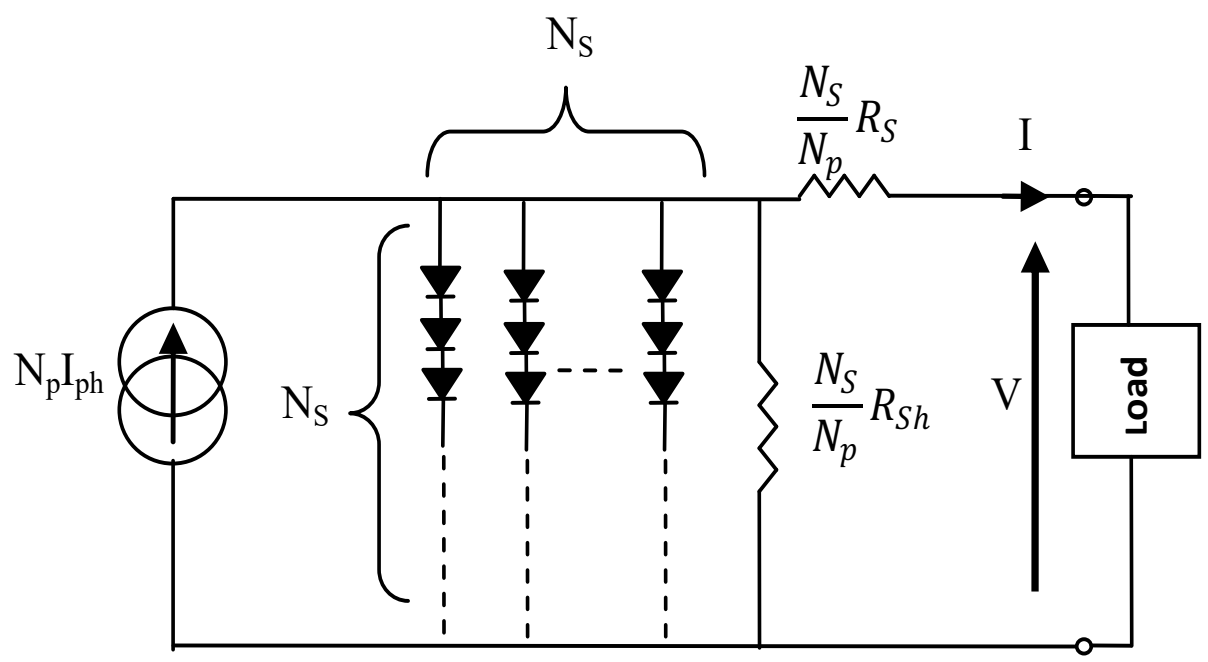

Figure 4. Single diode model of a PV array.

From the Figure 4, the voltage-current relationship of PV array is as follows:

$I=N_{p} I_{p h}-N_{p} I_{s}\left[\exp \left(\frac{q\left(V+I R_{S}\right)}{k T A}\right)-1\right]-\frac{V+I R_{S}}{R_{S h}}$

The parameters of the specified in PV model are shown in Table 1, and the module is made by $50 \mathrm{~W}$ singlecrystalline PV module SM50-H.

Table 1. PV Panel parameters.

\begin{tabular}{|l|c|c|}
\hline \multicolumn{1}{|c|}{ Parameter at STC } & Abbreviation & Value \\
\hline Band-gap energy & $\mathrm{E}_{\mathrm{G}}$ & $1.12 \mathrm{eV}$ \\
\hline Ideal factor & $\mathrm{A}$ & 1.5 \\
\hline Maximum power & $\mathrm{P}_{\max }$ & $50 \mathrm{Wp}$ \\
\hline Rated current & $\mathrm{I}_{\mathrm{MPP}}$ & $3.15 \mathrm{~A}$ \\
\hline Rated voltage & $\mathrm{V}_{\mathrm{MPP}}$ & $15.9 \mathrm{~V}$ \\
\hline Short circuit current & $\mathrm{I}_{\mathrm{sc}}$ & $3.35 \mathrm{~A}$ \\
\hline Open circuit voltage & $\mathrm{V}_{\mathrm{oc}}$ & $19.8 \mathrm{~V}$ \\
\hline Temperature coefficient of $\mathrm{I}_{\mathrm{sc}}$ & $\mathrm{K}_{\mathrm{i}}$ & $1.2 \mathrm{~mA} /{ }^{\circ} \mathrm{C}$ \\
\hline Cell serial modules & $\mathrm{n}_{\mathrm{s}}$ & 33 \\
\hline
\end{tabular}




\section{BOOST CONVERTER}

The power converter was inserted between the PV generator and the load in order to improve the performance of a photovoltaic system. The DC-DC converter "boost" shown in Figure 5, (Khiareddine et al. 2013), controlled by a tracking algorithm of MPPT (duty cycle $\alpha$ ), provides the proper voltage to the load. The MPPT drives the operating point of the PVP to the Pmax detected by the control system.

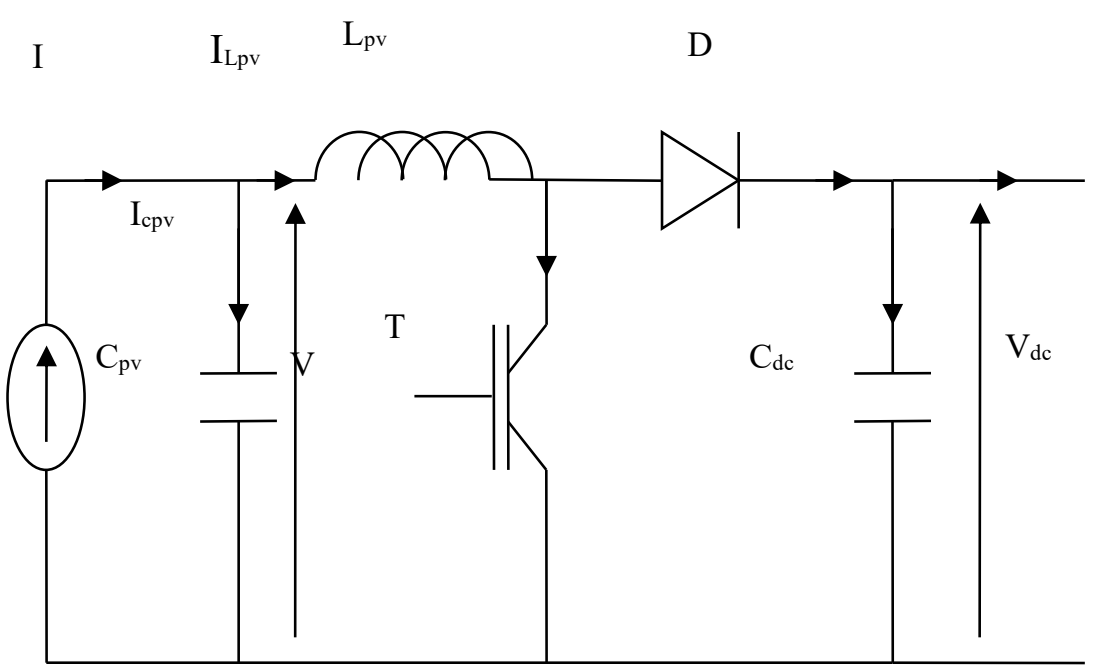

Figure 5. Equivalent circuit for Boost converter.

The mathematical model of the boost converter is given by the following equation:

$$
\left\{\begin{array}{l}
\frac{d V}{d t}=\frac{1}{C_{p v}}\left(I-I_{L p v}\right) \\
\frac{d I_{L p v}}{d t}=\frac{1}{L_{p v}}\left(V-\left(1-\alpha_{p v}\right) V_{d c}\right)
\end{array}\right.
$$

\section{BLOCK OF MPPT CONTROL}

This part is constituted by two stages. The first one is used to estimate the maximum power delivered by the photovoltaic panel (PVP). The second stage is used to estimate the optimal duty cycle used to control the boost converter, to make the PVP operate in its maximum power, in each solar radiation values (Sihem Amara et al. 2021).

\subsubsection{Estimation of Maximum Power}

The following equation has been established to estimate the maximum power, drawn by the PVP for all values of the solar radiation. This equation needs just the standard maximum power given by the builder at the back of the $\mathrm{PVP}\left(\mathrm{PM}\right.$ at $25^{\circ} \mathrm{C}$ and $\left.1000 \mathrm{~W} / \mathrm{m}^{2}\right)$ to estimate in real time the maximum power $\mathrm{P}_{\mathrm{M}}$ for each solar radiation change, in real time. 
$P_{M}=P_{M-S} \cdot \frac{G}{1000}$

\subsubsection{Neuro-Fuzzy Estimator}

ANFIS [1] implements a Takagi Sugeno FIS and has a five-layered architecture as shown in Figure 7. The first hidden layer is for fuzzification of the input variables, and T-norm operators are deployed in the second hidden layer to compute the rule antecedent part. The third hidden layer normalizes the rule strengths followed by the fourth hidden layer, where the consequent parameters of the rule are determined. Output layer computes the overall input as the summation of all incoming signals.

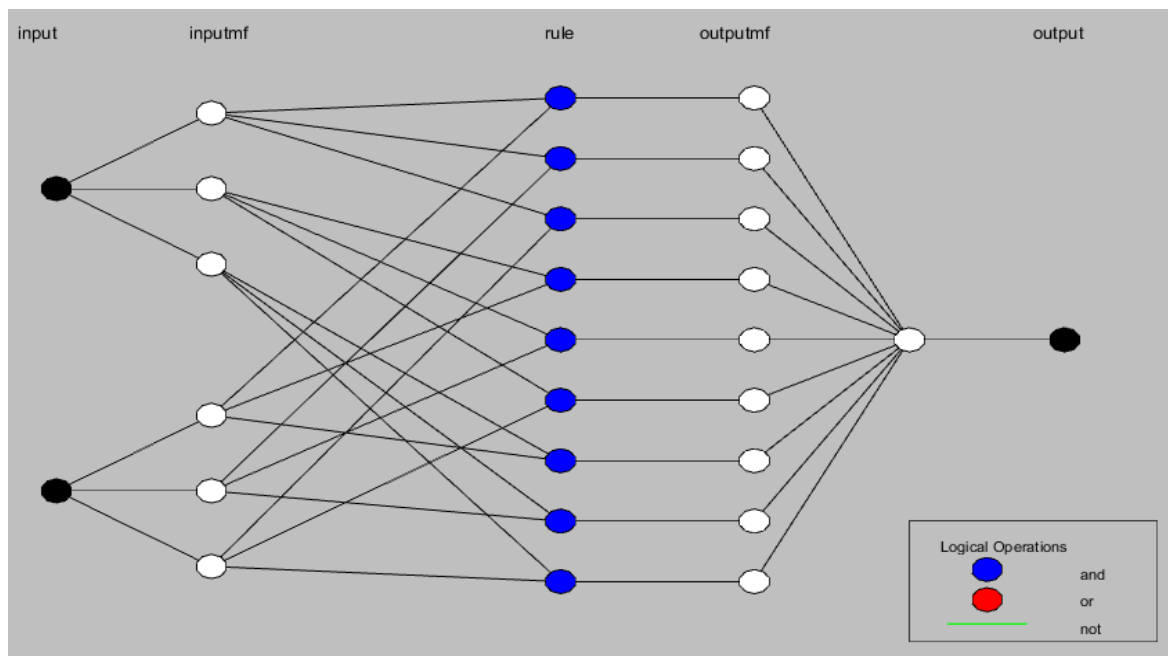

Figure 7. Structure of ANFIS.

\subsubsection{Estimation of Optimum Duty Cycle}

Figure 8 gives the used strategy to estimate, in real time, the optimal duty cycle, $\alpha_{o p}$, for all the solar radiation values.

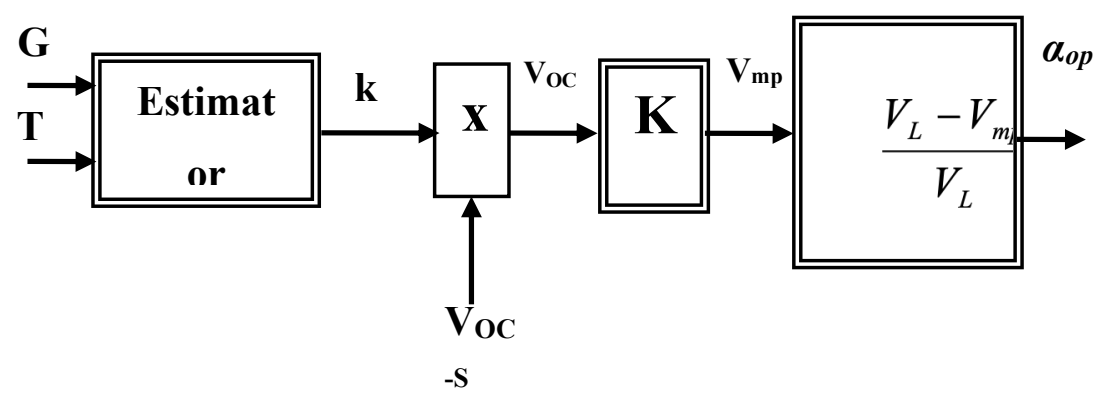

Figure 8. Optimum duty cycle estimation diagram. 
This strategy consists in developing a neurofuzzy estimator that can determine the ratio, $\mathrm{k}$, between the open circuit voltage, $\mathrm{V}_{\mathrm{OC}}$, and the standard open circuit voltage, $\mathrm{V}_{\mathrm{OC}-\mathrm{S}}$, for any solar radiation values $\mathrm{G}$.

$k=\frac{V_{O C}}{V_{O C-S}}$

This ratio will then be multiplied by the standard open circuit voltage (given by the builder at the back of the PVP) to give the value of the open circuit voltage for all the solar radiation values G.

Then, a new relationship between the voltage of the open circuit and the voltage corresponding to the maximum power, $\mathrm{V}_{\mathrm{mp}}$, for all values of solar radiation, has been established. This relationship has been found, based on the practical experiments given by Figure 9 .

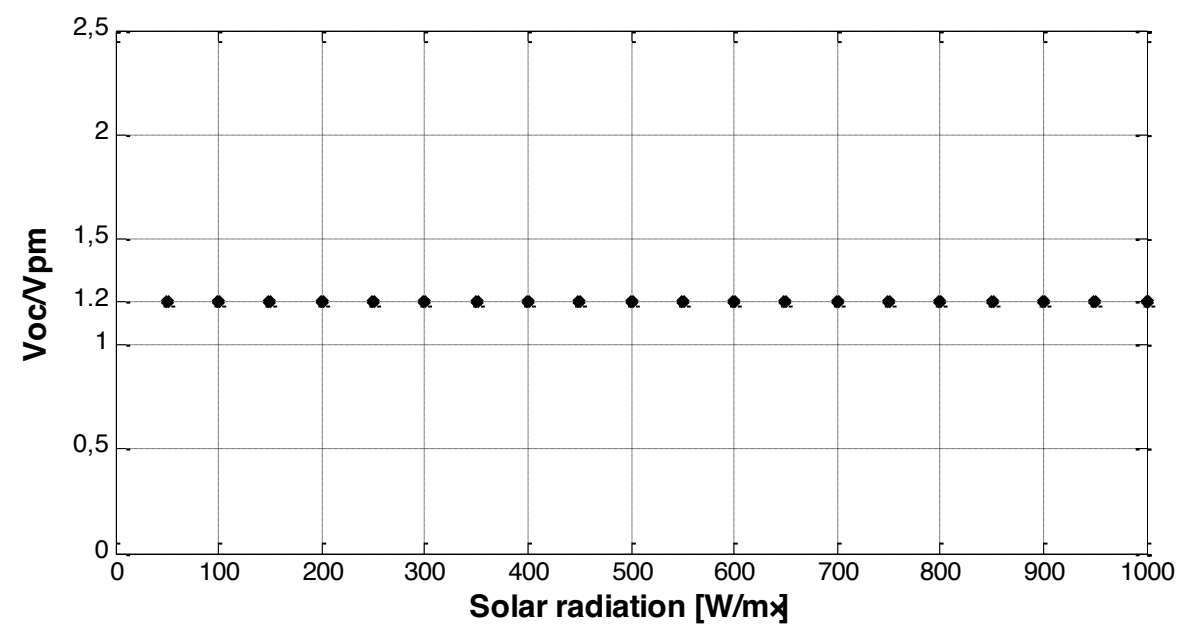

Figure 9. The relationship of the coefficient Voc/Vpm and the solar radiation.

Finally, the optimal duty cycle has been calculated directly from the voltage corresponding to the maximum power by the relation of the boost converter

$V_{L}=\frac{V_{m p}}{1-\alpha_{o p}}$

\section{SIMULATION STUDY}

Based on the system presented in Fig. 1 and MPPT controller methods, the photovoltaic supplying system with DC load (Battery of 24V) is modeled and simulated in a friendly user MATLAB/Simulink environment.

In order to check the MPPT performance under the different climatic conditions, some simulation studies have been carried out using the real solar radiation and the temperature. 


\section{Database Description}

Our proposed MPPT algorithm is based on a real database acquired by a measuring station installed at the Higher Institute of Applied Sciences and Technologies of Sousse-Tunisia (Figure6). This station is composed of an acquisition chain, which records the climatic parameters and the electrical parameters of the panel every $5 \mathrm{~min}$. This base is refined using advanced software MySQL.

Our system is based on a database that takes as inputs a real measured parameters: the solar radiation $G$, the cell temperature $T$, and it gives as output the voltage corresponding to the maximum power $V_{m p}$, the current corresponding to the maximum power $I_{m p}$, the maximum power $P_{m}$, the open circuit voltage $V_{o c}$, and the optimal duty cycle $\alpha_{o p}$.
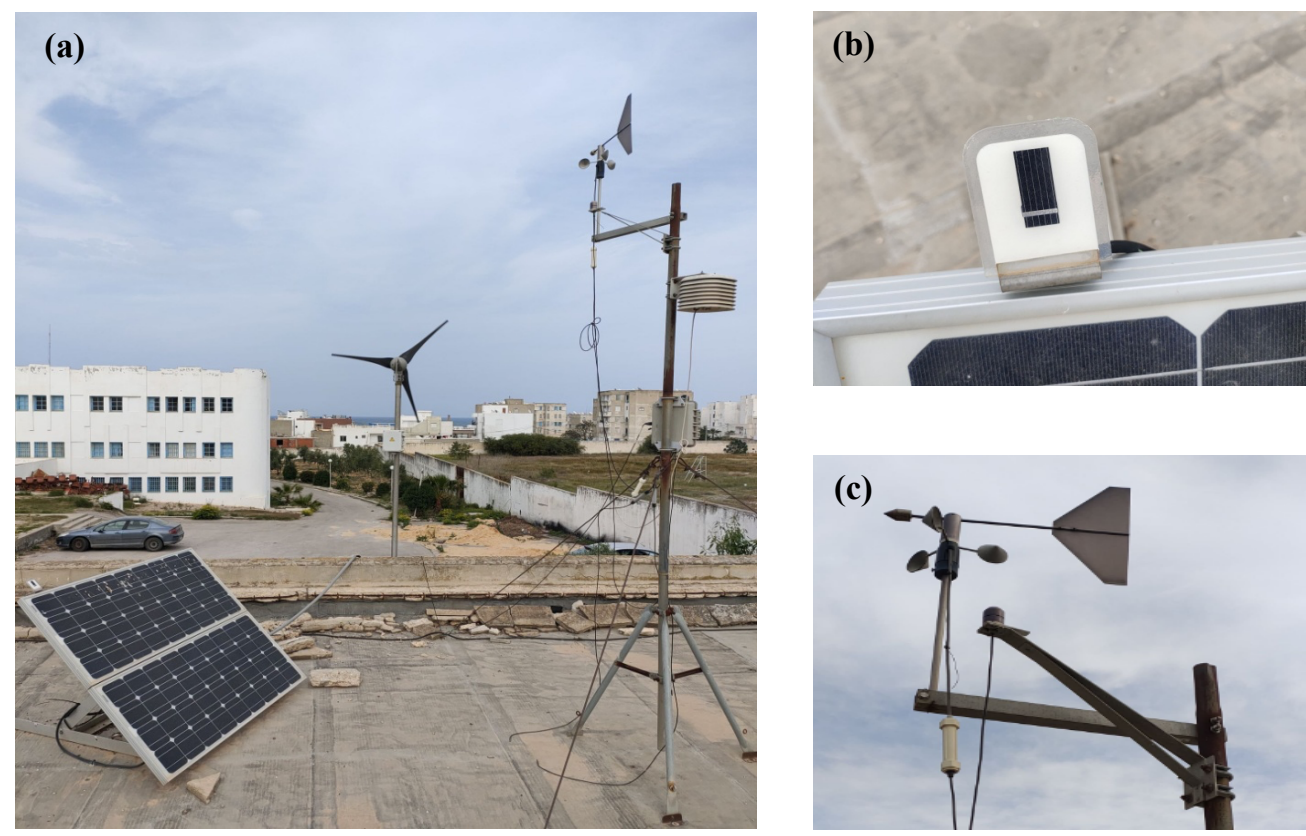

Figure 6. (a) Hybrid PVP/WT system; (b) Solar radiation sensor; (c) Wind sensor.

Fig 7 shows the flowchart for acquiring database parameters. For each couple $\mathrm{G}$ and $\mathrm{T}$, we vary a resistive charge from 0 to infinity, and we read the current and voltage electrical values of the panel displayed by measuring devices. 


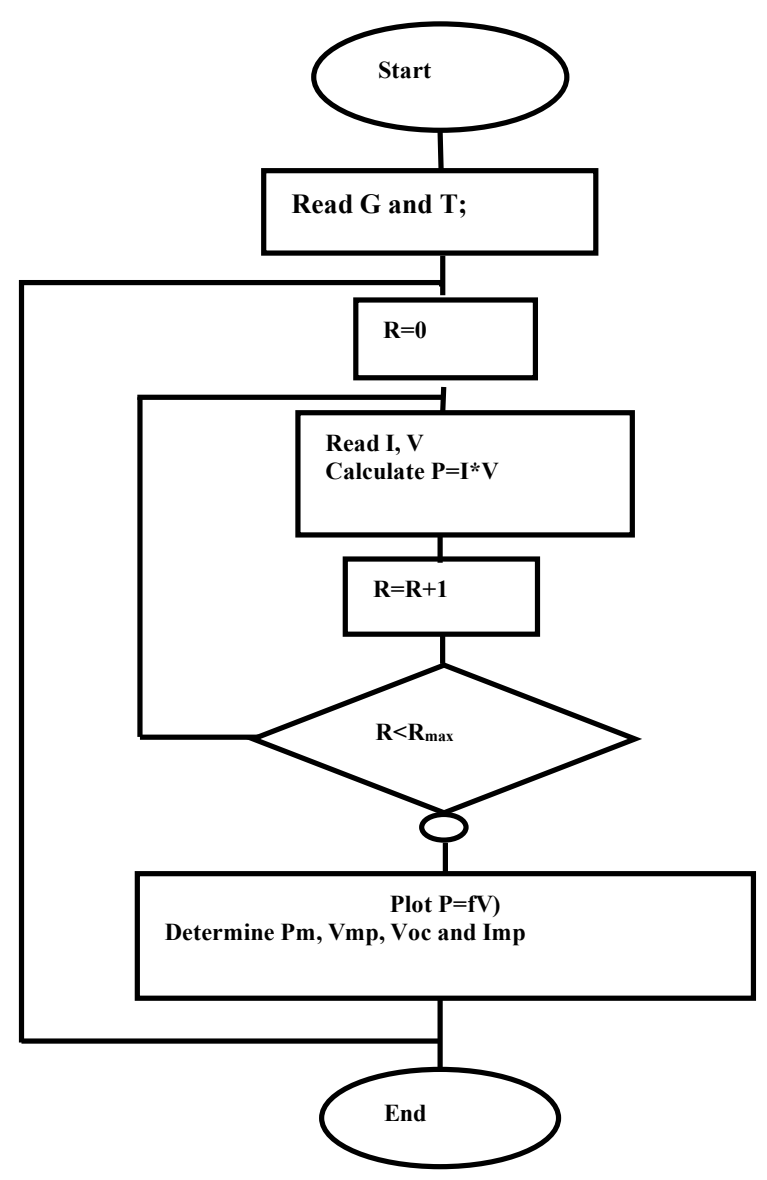

Figure 7. Data acquisition flowchart.

\section{Simulation Results}

To prove the efficiency of the neuro-fuzzy estimator, a comparison between the real and the estimated values of the ratio of the open circuit voltage and the standard value for different solar radiation has been developed (Figure $10)$.

Figure 10 gives an almost superposition of the actual and the estimated values of the ratio of the open circuit voltage and the standard value for different solar radiation. It is clear from Figure 10 that the neural estimator values follow suitably the real one. The error is estimated to be less than $2 \%$.

In a second step, based on the elaborated relationship between the maximum power voltage and the open circuit voltage of the PVP, we have presented the estimated and the real values of the maximum power voltage for the different solar radiation values (Figure 11). The estimated and the real values are proved to be almost confused with a normalized error less than $2 \%$.

In another step, we have shown in Figure 12 the real and the estimated duty cycle for the different solar radiation values. The figure, clearly, demonstrates the MPPT's effectiveness in a properly estimating in real time the optimum value of the duty cycle. The error between the real and the estimated values is around $1 \%$. 


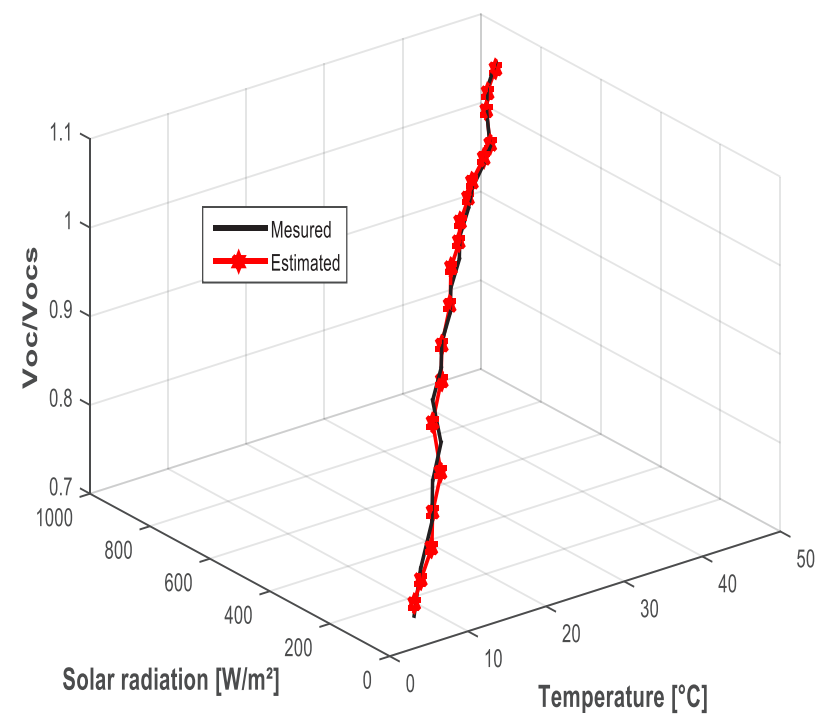

Figure 10. The evolution of the coefficient Voc/Vocs in function of climatic condition.

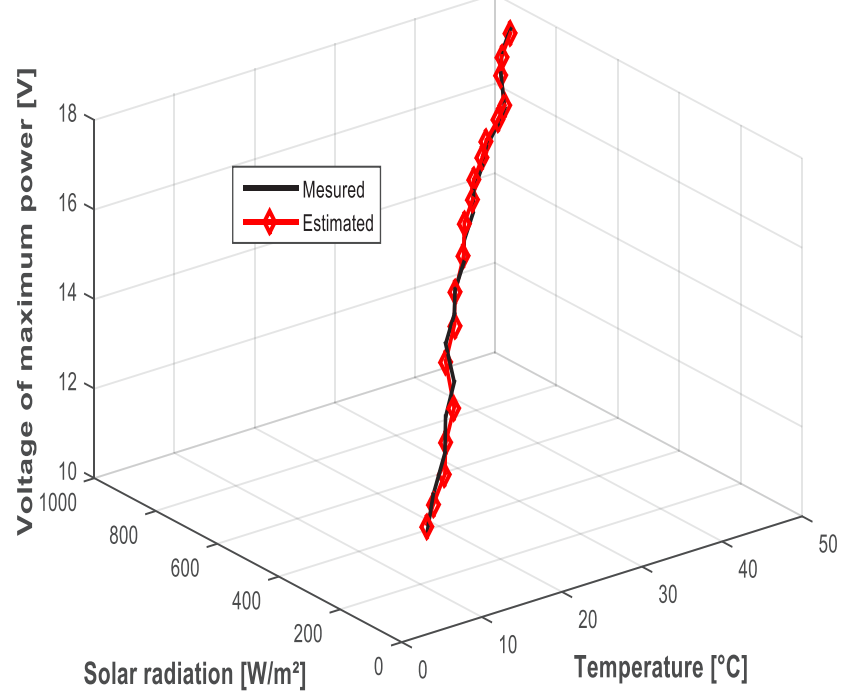

Figure 11. Real and estimated voltage of maximum power in function of climatic condition.

It is clear from Figure 13 that the PVP maximum power gained with the proposed MPPT controller is about $99 \%$ of the real maximum power. 
The performance of the MPPT can be detected, according to the efficiency (Ben Salah and Ouali, 2011). The efficiency is calculated by the following equation:

Efficiency $=\left(1-\frac{\operatorname{Re} \text { al } P_{\max }-\text { Estimate } P_{\max }}{\operatorname{Re} \text { al } P_{\max }}\right) 100$

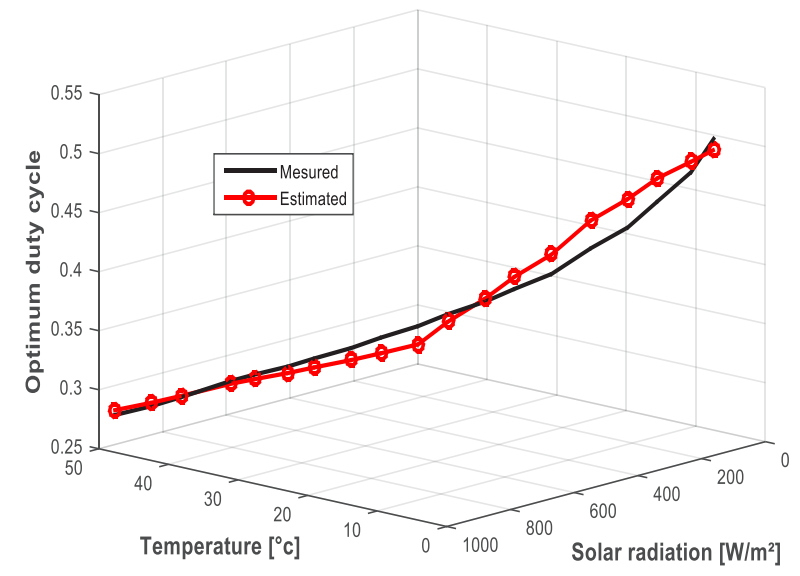

Figure 12. Variation of optimum duty cycle in function of climatic condition.

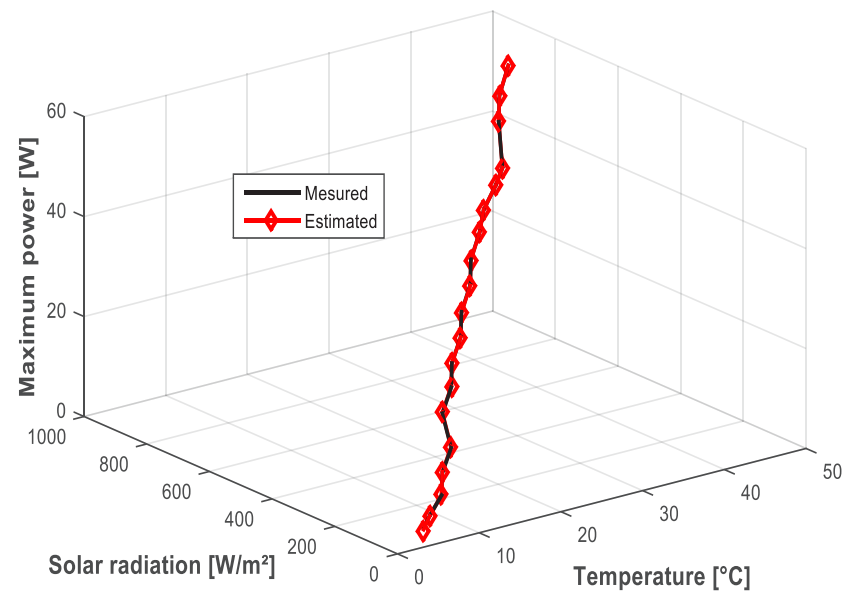

Figure 13. Variation of maximum power in function of climatic condition.

Table 2 gives the error estimates due to the proposal MPPT controller for the different PVP power. It shows also that the MPPT controller converges asymptotically to a real state. We can observe that when the precision increases, consequently, the error decreases when the PVP power increases. 
Table 2. Error estimates.

\begin{tabular}{|c|c|c|}
\hline & Neuro-Fuzzy & Neural Network \\
\hline PVP & Error (\%) & Error (\%) \\
\hline SM50-H & 0.18 & 1.4 \\
\hline SQ70 & 0.25 & 1.2 \\
\hline SK150W12V10 & 0.26 & 0.9 \\
\hline
\end{tabular}

\section{CONCLUSION}

This paper presented a new method for a maximum power point tracker (MPPT). The method consists in the development of the neurofuzzy estimator, which can determine, in real time, the value of the open circuit voltage for any climatic condition. A new relationship between the open circuit voltage, $\mathrm{V}_{\mathrm{OC}}$, and the voltage corresponding to the maximum power, $\mathrm{V}_{\mathrm{mp}}$, has been established. This $\mathrm{V}_{\mathrm{mp}}$ was used to calculate the optimal duty cycle. The simulation model was developed using MATLAB. The simulation studies were conducted to verify the performance of the system under the different climatic condition. The simulation results show the effectiveness of the proposed system. Improvements in Maximum power point tracking (MPPT) technologies are one of the most important aspects of constructing solar systems to boost output power generation. The adaptive neural fuzzy inference system (ANFIS) is one of the finest ways for finding the maximum power point (MPP) in solar systems among many methods because of its quick reaction and minimal fluctuations (Sudipta Saha et al. 2021). The suggested MPPT control approach has several advantages, including faster convergence to the maximum photovoltaic power, more efficiency than AI equivalents $(\mathrm{NN})$, resilience, ease of implementation, and the capacity to learn from prior data regardless of the season. As a result, the suggested algorithm, which outperforms the existing MPPT algorithms such as P\&O and IncCond, achieves considerably quicker convergence speeds.

Observations reveal that differences in input data or tiny fluctuations, as well as unpleasant and sudden weather changes, have no effect on the proposed method's performance or accuracy. With each repetition, the perturbation time reduces. At the end of each iteration, the suggested algorithm gradually learns and absorbs fresh data.

The proposed method keeps being simple, achievable, and practical and gives powers close to the real ones. Several conducted simulations have proved the performance of the system under different climatic condition and different PVP powers.

\section{ACKNOWLEDGMENT}

The authors extend their appreciation to the Deanship of Scientific Research at King Khalid University for funding this work through the Research Groups Program under grant number (RGP.1/299/42). 


\section{REFERENCES}

Abdelaziz Salah Saidi (2020). Impact of large photovoltaic power penetration on the voltage regulation and dynamic performance of the Tunisian power system. Energy Exploration \& Exploitation. 38 (5), pp. 17741809. https://doi.org/10.1177\%2F0144598720940864.

Abdelaziz Salah Saidi (2020). Investigation of Structural Voltage Stability in Tunisian Distribution Networks Integrating Large-Scale Solar Photovoltaic Power Plant. International Journal of Bifurcation and Chaos (IJBC).30 (13), pp. 1-24. https://doi.org/10.1142/S0218127420502594.

Abdelaziz Salah Saidi, Chokri Ben Salah, Ayachi Errachdi, Mohammad Fazle Azeem, Javed Khan Bhutto\& V.P.Thafasal Ijyas (2021). A novel approach in stand-alone photovoltaic system using MPPT controllers \& NNE. Ain Shams Engineering Journal Volume 12, Issue 2, June 2021, Pages 1973-1984.

Avila, L., De Paula, M., Trimboli, M., \& Carlucho, I. (2020). Deep reinforcement learning approach for MPPT control of partially shaded PV systems in Smart Grids. Applied Soft Computing, 106711.

Dandoussou A, Martin K, Laurent B, et al. (2016). Comparative study of the reliability of MPPT algorithms for the crystalline silicon photovoltaic modules invariable weather conditions. Journal of Electrical Systems and Information Technology. (117):1-12.

Dousoky GM and Masahito S (2017). New parameter for current-sensorless MPPT in grid-connected photovoltaic VSIs. Solar Energy, Elsevier (143): 113-119.

Ferdaws Ben Naceur, Chokri Ben Salah, Achraf Jabeur Telmoudi and Mohamed Ali Mahjoub (2021). Intelligent approach for optimal sizing in photovoltaic panel-battery system and optimizing smart grid energy. Transactions of the Institute of Measurement and Control 1-17.

Ferdaws Ben Naceur, Achraf Jabeur Telmoudi and Mohamed Ali Mahjoub (2020). A proposal ANFIS estimation algorithm for optimal sizing of a PVP/Battery system. In: 7th International Conference on Control, Decision and Information Technologies (CoDIT) 29 June-2 July 2020, Prague, Czech Republic.

Jiang JA, Su YL, Kuo KC, et al. (2017). On a hybrid MPPT control scheme to improve energy harvesting performance of traditional two-stage inverters used in photovoltaic systems. Renewable and Sustainable Energy Reviews. (69): 1113-1128.

Sihem Amara, Sana Toumi, Chokri Ben Salah, et al. (2021). Improvement of Techno-Economic Optimal Sizing of a Hybrid off-grid Micro-grid System. Energy. ELSEVIER-Science Direct. (233). https://doi.org/10.1016/j.energy.2021.121166

Lulian M and Antoneta LB (2015). MPPT for grid-connected photovoltaic systems using ripple-based Extremum Seeking Control: Analysis and control design issues. Solar Energy, Elsevier. (111): 30-42.

Mahmoud A. Soliman, Hany M. Hasanien and Abdulaziz Alkuhayl (2020). Marine predators algorithm for parameters identification of triple-diode photovoltaic models. IEEE ACCESS.8, pp. 155832-155842.

Mohammed Qais, Hany M. Hasanien and Saad Alghuwainem (2020). Transient search optimization for electrical parameters estimation of photovoltaic module based on datasheet values. Energy Conversion and Management. 214, 112904, pp. 1-9.

Nouhaila Lazaar, Eyman Fakhri, Mahmoud Barakat, Jalal Sabor and Hamid Gualous (2021). A genetic algorithm based optimal sizing strategy for PV/battery/hydrogen hybrid system. In: Masrour T, El Hassani I and Cherrafi A (eds) Artificial Intelligence and Industrial Applications. A2IA 2020. Lecture Notes in Networks and Systems Volume 144, Springer, Cham, pp. 247-259.

Omnia S. Elazab, Mahdi Debouza, Hany M. Hasanien, S.M. Muyeen, Ahmed Al-Durra (2020). Salp swarm algorithm-based optimal control scheme for LVRT capability improvement of grid-connected photovoltaic power plants: design and experimental validation. IET Renewable Power Generation. 14 (4), pp. 591-599. 
Osisioma E, Fu Z and Li Z (2017). Energy Performance and Cost Comparison of MPPT Techniques for Photovoltaics and other Applications. Energy Procedia (107): 297-303.

Rahmani B, Weixing L and Guihua L (2015). An Advanced Universal Power Quality Conditioning System and MPPT method for grid integration of photovoltaic systems. Electrical Power and Energy Systems (69): 7684.

Sachin VR and Vivek JP (2016). Experimental verification of rate of charge improvement using photovoltaic MPPT hardware for battery and ultra-capacitor storage devices. Solar Energy, Elsevier (139): 142-148.

Salman S, Xin AI and Zhouyang WU (2018). Design of a P-\&-O algorithm based MPPT charge controller for a stand-alone 200W PV system. Protection and Control of Modern Power Systems 3(25).

Saidi A and Helmy W (2019). Artificial neural network-aided technique for low voltage ride-through wind turbines for controlling the dynamic behavior under different load conditions. Wind Engineering Journal 43(4): 420-440.

Sellami A, Khalid K, Rabie O, et al. (2018). A Novel Auto-Scaling MPPT Algorithm based on Perturb and Observe Method for Photovoltaic Modules under Partial Shading Conditions. Applied Solar Energy 54 (3): $149-158$.

Sudipta Saha; Syed Muhammad Amrr; Abdelaziz Salah Saidi; Arunava Banerjee; M. Nabi (2021). FiniteTime Adaptive Higher-Order SMC for the Nonlinear Five DOF Active Magnetic Bearing System. Electronics. 10(11), https://doi.org/10.3390/electronics10111333 